\title{
Evolution in Sinonasal Mucosal Melanoma Management
}

\author{
Tony Richa ${ }^{1}$ Alice Lee ${ }^{1} \quad$ Marc A. Cohen $^{1}$ \\ ${ }^{1}$ Department of Head and Neck Surgery, Memorial Sloan Kettering \\ Cancer Center, New York, New York, United States \\ Address for correspondence Tony Richa, MD, Department of Head \\ and Neck Surgery, Memorial Sloan Kettering Cancer Center, 1275 York \\ Avenue, New York, NY 10065-6007, United States \\ J Neurol Surg Rep 2022;83:e1-e2. \\ (e-mail: tonyricha88@gmail.com).
}

\begin{abstract}
Sinonasal mucosal melanoma is a rare and aggressive cancer with poor prognosis. Surgical resection with clear margins, when possible, remains the treatment of choice.

Keywords

- melanoma

- sinonasal mucosal melanoma

- immunotherapy

- management

- review Radiation therapy is generally used in the adjuvant setting with improved rates of local control following complete resection. Traditional chemotherapeutic agents do not improve the rates of locoregional control or survival. Immunotherapy has been used with some responders but with overall relatively poor outcomes. These outcomes highlight the need for new agents and more prospective trials in this space. We provide a unique case report of a patient with an advanced sinonasal mucosal melanoma and an overview of the recent literature pertaining to the management of this disease.
\end{abstract}

\section{Introduction}

Sinonasal mucosal melanomas are rare with most presenting with locally advanced disease. ${ }^{1,2}$ Traditionally, management relied on radical resection followed by radiation therapy. Regardless of local treatment, outcomes are historically poor secondary to high rates of distant metastasis., ${ }^{3,4}$ With emergence of immune checkpoint inhibition (ICI) and a focus on quality of life, management of this disease has evolved.

\section{Case Review}

A 79-year-old male presented with left nasal obstruction, epistaxis, and proptosis. A magnetic resonance imaging demonstrated a multicompartmental sinonasal mass that measured $6.8 \times 4.9 \times 3.8 \mathrm{~cm}$ with invasion of the inferior rectus muscle and orbital apex ( - Fig. 1A, B). Biopsy demonstrated mucosal melanoma. Following progression after radiation therapy (30 Gy) and PD-1 blockade (nivolumab), the patient then received combined programmed cell death protein 1 (PD-1) blockade with cytotoxic T-lymphocyteassociated protein 4 (CTLA-4) inhibition (ipilimumab). The patient had a complete response after only one dose ( - Fig. 1C, D). The patient developed treatment-related toxicities including diabetes, hypothyroidism, and autoimmune ocular disease, and unfortunately died 18 months after treatment due to a pulmonary embolus.

\section{Literature Review}

Head and neck mucosal melanoma carries a 5-year overall survival (OS) of $20 \% .^{5}$ Radical resection of sinonasal malignant melanoma (SNMM) with clear margins can entail severe cosmetic and functional impairments. ${ }^{6}$ In a large retrospective study, positive margins were noted in $22 \%$ of patients demonstrating some of the biological challenges of this disease. ${ }^{5}$ Open craniofacial resection is no longer recommended due to poor outcomes and associated morbidity. ${ }^{7}$ Endoscopic resection has been shown to have lower morbidity, better quality of life, and comparable survival outcomes to open surgery. ${ }^{3,8,9}$ Adjuvant radiation therapy has been shown to improve local control but does not seem to improve survival. ${ }^{10,11}$ received

September 10, 2021

accepted after revision

November 8, 2021
DOI https://doi.org/

$10.1055 / \mathrm{s}-0041-1741380$.

ISSN 2193-6358.

\footnotetext{
(C) 2022. The Author(s).

This is an open access article published by Thieme under the terms of the Creative Commons Attribution-NonDerivative-NonCommercial-License, permitting copying and reproduction so long as the original work is given appropriate credit. Contents may not be used for commercial purposes, or adapted, remixed, transformed or built upon. (https://creativecommons.org/ licenses/by-nc-nd/4.0/) Georg Thieme Verlag KG, Rüdigerstraße 14, 70469 Stuttgart, Germany
} 

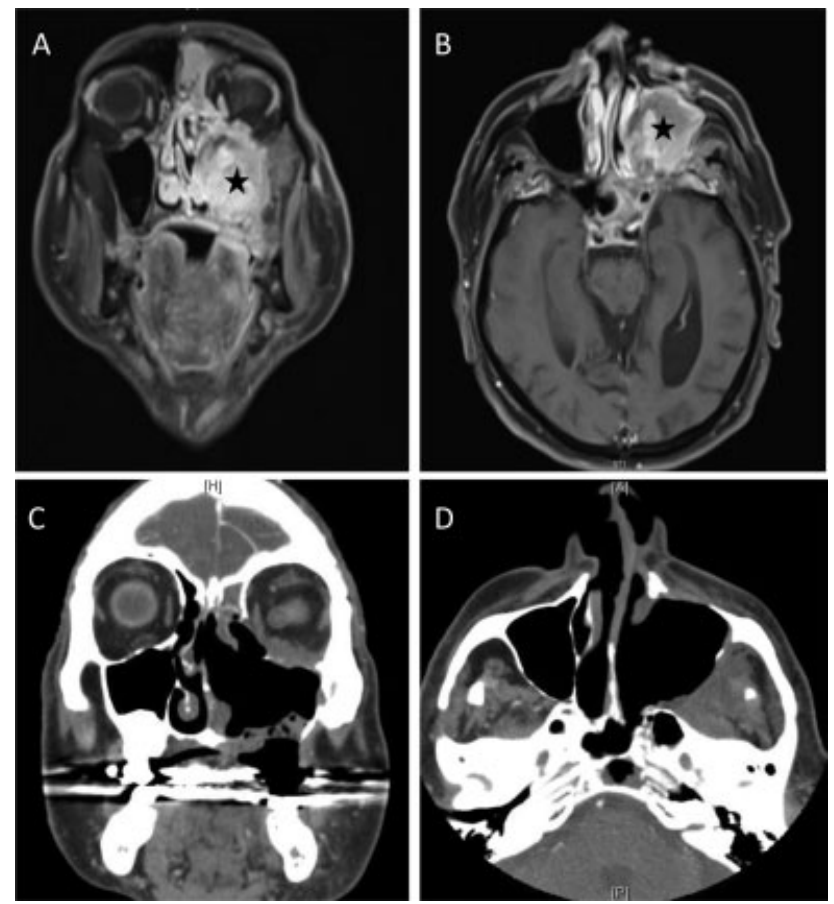

Fig. 1 Pretreatment coronal (A) and axial (B) magnetic resonance imaging scans of left sinonasal malignant melanoma. Posttreatment images represent complete response of the tumor to combination immune checkpoint inhibition on coronal (C) and axial (D) computed tomographic scans.

In contrast to cutaneous melanoma, SNMM is notable for a lack of BRAF mutations (0-3\%) and a high incidence of c-KIT (4-53\%) and NRAS (14-48\%) mutations. ${ }^{12,13}$ Studies of the tyrosine kinase inhibitor imatinib mesylate in select cases demonstrated an overall response (OR) of 16 to $29 \% .^{14,15}$ For SNMM with NRAS mutations, mitogen-activated protein kinase inhibitors have been trialed with modest responses and questionable clinical benefit. ${ }^{16,17}$ With a lower response rate than cutaneous melanoma, ipilimumab and pembrolizumab monotherapy for mucosal melanomas was shown to have an OR of 6.7 and $23 \%$, respectively. ${ }^{18,19}$ In another study comparing combination PD- 1 and CTLA-4 inhibition versus monotherapy in mucosal melanoma, OR was found to be $37 \%$ ( $3 \%$ complete response) compared with $23 \%$ (nivolumab) and $8 \%$ (ipilimumab). ${ }^{20}$ However, grade 3 and 4 adverse events were significantly higher in the combination group (40 vs. $8 \%)$.

In a single-institution retrospective study assessing outcomes over time with integration of endoscopic surgery, advances in radiation, and immunotherapy, there have been no changes in overall survival. ${ }^{4}$ Modern management relies on ICI, but the appropriate timing of integration is unclear. Future studies are needed to analyze the impact of new agents and protocols on survival outcomes and quality of life.

\section{Conflict of Interest}

None.

\section{References}

1 Chang AE, Karnell LH, Menck HRThe American College of Surgeons Commission on Cancer and the American Cancer Society. The National Cancer Data Base report on cutaneous and noncutaneous melanoma: a summary of 84,836 cases from the past decade. Cancer 1998;83(08):1664-1678

2 Bachar G, Loh KS, O'Sullivan B, et al. Mucosal melanomas of the head and neck: experience of the Princess Margaret Hospital. Head Neck 2008;30(10):1325-1331

3 Amit M, Tam S, Abdelmeguid AS, et al. Patterns of treatment failure in patients with sinonasal mucosal melanoma. Ann Surg Oncol 2018;25(06):1723-1729

4 Flukes S, Lohia S, Barker CA, et al. Are our patients doing better? A single institution experience of an evolving management paradigm for sinonasal mucosal melanoma. Oral Oncol 2021;112:105006

5 Ascierto PA, Accorona R, Botti G, et al. Mucosal melanoma of the head and neck. Crit Rev Oncol Hematol 2017;112:136-152

6 Lazarev S, Gupta V, Hu K, Harrison LB, Bakst R. Mucosal melanoma of the head and neck: a systematic review of the literature. Int J Radiat Oncol Biol Phys 2014;90(05):1108-1118

7 Ganly I, Patel SG, Singh B, et al. Craniofacial resection for malignant melanoma of the skull base: report of an international collaborative study. Arch Otolaryngol Head Neck Surg 2006;132 (01):73-78

8 Sayed Z, Migliacci JC, Cracchiolo JR, et al. Association of surgical approach and margin status with oncologic outcomes following gross total resection for sinonasal melanoma. JAMA Otolaryngol Head Neck Surg 2017;143(12):1220-1227

9 Lund VJ. Sinonasal Malignant Melanoma. Adv Otorhinolaryngol 2020;84:185-196

10 Samstein RM, Carvajal RD, Postow MA, et al. Localized sinonasal mucosal melanoma: outcomes and associations with stage, radiotherapy, and positron emission tomography response. Head Neck 2016;38(09):1310-1317

11 Krengli M, Jereczek-Fossa BA, Kaanders JHAM, Masini L, Beldì D, Orecchia $R$. What is the role of radiotherapy in the treatment of mucosal melanoma of the head and neck? Crit Rev Oncol Hematol 2008;65(02):121-128

12 Mikkelsen LH, Larsen AC, von Buchwald C, Drzewiecki KT, Prause JU, Heegaard S. Mucosal malignant melanoma - a clinical, oncological, pathological and genetic survey. APMIS 2016;124(06):475-486

13 Öztürk Sari Ş, Yilmaz İ, Taşkin OÇ, et al. BRAF, NRAS, KIT, TERT, GNAQ/GNA11 mutation profile analysis of head and neck mucosal melanomas: a study of 42 cases. Pathology 2017;49(01):55-61

14 Carvajal RD, Antonescu CR, Wolchok JD, et al. KIT as a therapeutic target in metastatic melanoma. JAMA 2011;305(22):2327-2334

15 Guo J, Si L, Kong Y, et al. Phase II, open-label, single-arm trial of imatinib mesylate in patients with metastatic melanoma harboring cKit mutation or amplification. J Clin Oncol 2011;29(21):2904-2909

16 Ascierto PA, Schadendorf D, Berking C, et al. MEK162 for patients with advanced melanoma harbouring NRAS or Val600 BRAF mutations: a non-randomised, open-label phase 2 study. Lancet Oncol 2013;14(03):249-256

17 Dummer R, Schadendorf D, Ascierto PA, et al. Binimetinib versus dacarbazine in patients with advanced NRAS-mutant melanoma (NEMO): a multicentre, open-label, randomised, phase 3 trial. Lancet Oncol 2017;18(04):435-445

18 Postow MA, Luke JJ, Bluth MJ, et al. Ipilimumab for patients with advanced mucosal melanoma. Oncologist 2013;18(06):726-732

19 Shoushtari AN, Munhoz RR, Kuk D, et al. The efficacy of anti-PD-1 agents in acral and mucosal melanoma. Cancer 2016;122(21): 3354-3362

20 D'Angelo SP, Larkin J, Sosman JA, et al. Efficacy and safety of nivolumab alone or in combination with ipilimumab in patients with mucosal melanoma: A pooled analysis. J Clin Oncol 2017;35 (02):226-235 